\title{
SEMI-COMPACT SPACES AND DIMENSION
}

\author{
TOGO NISHIURA
}

All spaces under discussion will be separable and metrizable. $X$ is called semi-compact if each point of $X$ has arbitrary small neighborhoods with compact boundary.

In [dG] J. de Groot shows that a semi-compact space $X$ can be compactified in such a manner that the set of points added to $X$ is of dimension no larger than zero. Furthermore, any subspace $X$ of a compact space $\bar{X}$ such that $\bar{X} \backslash X$ is of dimension zero is semicompact. H. Freudenthal shows that a semi-compact space for which the space of quasicomponents is compact has an end-point compactification. The set of points added in the Freudenthal compactification is zero-dimensional $[\mathbf{F} 1 ; \mathbf{F} 2]$. Let us call any compactification of a space formed by adding an $n$-dimensional set an $n$-compactification. Then every semi-compact space has a zero-compactification. Also, the Freudenthal compactification mentioned above is a zerocompactification.

It is well known that $X$ can be compactified so that the compactification is of the same dimension as $X$ [HW]. J. de Groot posed the following question: "Does every semi-compact space have a dimension-preserving zero-compactification?" In other words, what is the relationship between zero-compactifications and dimension-preserving compactifications? This paper shows that there are semi-compact spaces $X$ with the property that every zero-compactification of $X$ is of higher dimension than $X$. Furthermore, there are connected spaces $X$ with the above mentioned property. Hence, the Freudenthal compactification of $X$ is of higher dimension than $X$.

1. Dimension theory. The following theorem is true.

Theorem. Let $X$ be a space and $M$ a subset such that $\operatorname{dim} X=n$ $(<+\infty), \operatorname{dim} M=m$ and $M$ is $F_{\sigma}$. Then for two disjoint closed sets $A_{1}$ and $A_{2}$ of $X$, there is a closed set $C$ such that $C$ separates $A_{1}$ and $A_{2}$, $\operatorname{dim} C \leqq n-1$ and $\operatorname{dim} C \cap M \leqq m-1$.

To prove this theorem the following lemma is needed.

Lemma. Suppose $\operatorname{dim} X=n(<+\infty)$. Then $X$ is the union of $n+1$ mutually disjoint zero-dimensional subsets, one of which is $F_{\sigma}$.

Proof. This lemma follows easily from [HW, p. 30]. The only fact one needs is that an $F_{\sigma}$ set of an $F_{\sigma}$ set is also $F_{\sigma}$.

Received by the editors November 18, 1960. 
Proof OF THEOREM. From the lemma we deduce that $X=X_{0} \cup X_{n-1}$ where $X_{0} \cap X_{n-1}=\varnothing, \operatorname{dim} X_{0}=0, \operatorname{dim} X_{n-1} \leqq n-1$ and $X_{0}$ is an $F_{\sigma}$ set in $X$. Also, since $M$ is $F_{\sigma}$ and $\operatorname{dim} M=m$, we deduce that $M=M_{0}$ $\cup M_{m-1}$ where $M_{0} \cap M_{m-1}=\varnothing, \operatorname{dim} M_{m-1} \leqq m-1$ and $M_{0}$ is an $F_{\sigma}$ set in $X$. Now, $\operatorname{dim}\left(M_{0} \cup X_{0}\right)=0$ since both $X_{0}$ and $M_{0}$ are zerodimensional and $F_{\sigma}$ [HW, Theorem II 2]. By virtue of [HW, 5B, p. 34], we have that there is a closed set $C$ such that $C$ separates $A_{1}$ and $A_{2}$ and $C \cap\left(M_{0} \cup X_{0}\right)=\varnothing$. Since $C \subset X_{n-1}$, we have $\operatorname{dim} C$ $\leqq n-1$. Also, since $C \cap M \subset M_{m-1}$, we have $\operatorname{dim} C \cap M \leqq m-1$. This concludes the proof of the theorem.

2. Example. We now exhibit a connected semi-compact space $X$ for which every zero-compactification is of higher dimension than $X$.

Let $n$ be a positive integer. $I^{n+1}$ will denote the product of $n+1$ closed unit intervals and $R$ will denote the countable set of points of $I^{n+1}$ all of whose coordinates are rational numbers.

Let $X=I^{n+1} \backslash R$. Then $X$ is semi-compact, $\operatorname{dim} X=n$ [HW, p. 29] and $X$ is an absolute $G_{\delta}[\mathbf{K}$, p. 207]. Let $\bar{X}$ be any zero-compactification of $X$. Then $\operatorname{dim}(\bar{X} \backslash X)=0$ and $\bar{X} \backslash X$ is an $F_{\sigma}$ set. Suppose $\operatorname{dim} \bar{X}$ $=n$ and let $x_{1}$ and $x_{2}$ be two distinct points of $X$. Then by the theorem above, there is a closed set $C$ which separates $x_{1}$ and $x_{2}, \operatorname{dim} C \leqq n-1$ and $\operatorname{dim}[C \cap(\bar{X} \backslash X)]=-1$. I.e., $C \cap(\bar{X} \backslash X)=\varnothing$. Clearly, $C$ is compact and $C \subset X$. By the sum theorem of dimension [HW, Theorem III 2], $\operatorname{dim}(C \cup R) \leqq n-1$. Also, $X \backslash C=I^{n+1} \backslash(C \cup R)$. By [HW, Corollary 2, p. 48] $I^{n+1} \backslash(C \cup R)$ is connected. Hence $C$ does not separate $x_{1}$ and $x_{2}$, a contradiction. Therefore $\operatorname{dim} \bar{X}=n+1$, and the example is exhibited.

Note added in proof. In the above example, the case $n=1$ has been essentially treated by $\mathrm{L}$. Zippin in $[\boldsymbol{Z}]$. Zippin shows that the Freudenthal end-point compactification in this case is two-dimensional. The maximal property of the end-point compactification $[F 2]$ together with the fact that closed, light mappings do not lower dimension imply that every zero-compactification in this case is twodimensional. The proof of the present paper is different from that of Zippin's. In fact, the proof can be extended to give the following result: Let $X$ satisfy the following conditions. (i) $\operatorname{dim} X=n \geqq 1$. (ii) $X$ is an absolute $G_{\delta}$. (iii) There is an $n+1$ Cantor manifold $Y$ such that $Y \supset X$ and $\operatorname{dim} Y \backslash X=0$. Then, $X$ is semi-compact and every zero-compactification is not a dimension-preserving compactification.

\section{BIBLIOGRAPHY}

[dG] J. de Groot, Topologische Studien, Groningen, Noordhoff, 1942. 
[F1] H. Freudenthal, Neuaufbau der Endentheorie, Ann. of Math. vol. 43, no. 2 (1942) pp. 261-279.

[F2] - Über die Enden diskreter Räume und Gruppen, Comment. Math. Helv. vol. 17 (1944-1945) pp. 1-38.

[HW] W. Hurewicz and H. Wallman, Dimension theory, Princeton, N. J., Princeton University Press, 1948.

[K] J. L. Kelley, General topology, New York, Van Nostrand, 1955.

[Z] L. Zippin, On semicompact spaces, Amer. J. Math. vol. 57 (1935) pp. 327-341.

University of Wisconsin, MilwaukeE

\section{ERGODIC AND MIXING PROPERTIES OF INFINITE MEMORY CHANNELS ${ }^{1}$}

\section{ROY L. ADLER}

1. Introduction. A. I. Khinchin [5] states that if an ergodic message space is fed into a channel with finite memory then the output message space is ergodic along with the compound message space of the in put with the output. However, Khinchin's notion of finite memory, definition (1) below, is apparently insufficient to yield this result. $\mathrm{K}$. Takano [6] has been able to establish it by strengthening the definition of finite memory to include both (1) and (2) below. The essential requirement for this theorem, however, is really (3) of which (2) is a special case. Condition (3) expresses that the output of a channel be asymptotically independent from the remote past of the input. The method of proof is an application of a functional form of the notion of ergodicity involving Cesàro convergence of a certain sequence of integrals. In addition this technique can be used to discuss some of the mixing and ergodic properties of the output with respect to the input and the channel.

2. Notation and Definitions. ${ }^{2}$ Let $(X, \mathfrak{X})$ be a measurable space with $X$ a space of points and $\mathfrak{X}$ a sigma-field of measurable subsets of $X$. Usually in information theory $X$ is referred to as an alphabet and is a finite set of points. We shall make no such restriction here. Con-

Received by the editors August 11, 1960 and, in revised form, December 9, 1960.

${ }^{1}$ Part of this work was supported by Air Force Contract No. SAR AF-49(638)-224 while the author was at Yale University.

${ }^{2}$ For complete definitions of concepts and standard results of measure theory and ergodic theory consult $[2 ; 3]$ respectively. 